\title{
GENERALIZATIONS OF BERNOULLI NUMBERS AND POLYNOMIALS
}

\author{
QIU-MING LUO, BAI-NI GUO, FENG QI, \\ and LOKENATH DEBNATH
}

Received 3 December 2001

\begin{abstract}
The concepts of Bernoulli numbers $B_{n}$, Bernoulli polynomials $B_{n}(x)$, and the generalized Bernoulli numbers $B_{n}(a, b)$ are generalized to the one $B_{n}(x ; a, b, c)$ which is called the generalized Bernoulli polynomials depending on three positive real parameters. Numerous properties of these polynomials and some relationships between $B_{n}, B_{n}(x), B_{n}(a, b)$, and $B_{n}(x ; a, b, c)$ are established.
\end{abstract}

2000 Mathematics Subject Classification: 11B68, 33E20.

1. Introduction. It is well known that Bernoulli's numbers and polynomials play important roles in mathematics. They are main objects in the theory of special functions [5]. Their definitions can be given as follows.

Definition 1.1. The numbers $B_{n}, 0 \leq n \leq \infty$, are called Bernoulli numbers if

$$
\phi(t)=\frac{t}{e^{t}-1}=\sum_{n=0}^{\infty} \frac{B_{n}}{n !} t^{n}, \quad|t|<2 \pi .
$$

Definition 1.2. The functions $B_{n}(x), 0 \leq n \leq \infty$, are called Bernoulli polynomials if they satisfy

$$
\phi(x ; t)=\frac{t e^{x t}}{e^{t}-1}=\sum_{n=0}^{\infty} \frac{B_{n}(x)}{n !} t^{n}, \quad|t|<2 \pi, x \in \mathbb{R} .
$$

The usual definition of higher-order Bernoulli polynomials is

$$
\frac{t^{\sigma} e^{u t}}{\left(e^{t}-1\right)^{\sigma}}=\sum_{n=0}^{\infty} \frac{B_{n}^{\sigma}(u)}{n !} t^{n}, \quad|t|<2 \pi .
$$

In $[2,4]$ the second and third authors generalized the concept of Bernoulli numbers as follows. 
DEFINITION 1.3. Let $a, b>0$ and $a \neq b$. The generalized Bernoulli numbers $B_{n}(a, b)$ are defined by

$$
\phi(t ; a, b)=\frac{t}{b^{t}-a^{t}}=\sum_{n=0}^{\infty} \frac{B_{n}(a, b)}{n !} t^{n}, \quad|t|<\frac{2 \pi}{|\ln b-\ln a|} .
$$

Among other things, some basic properties and relationships between $B_{n}$, $B_{n}(x)$, and $B_{n}(a, b)$ were also studied in $[2,4]$ initially and originally.

In this note, we first give definitions of the generalized Bernoulli polynomials, which generalize the concepts stated above, and then research their basic properties and relationships with Bernoulli numbers $B_{n}$, Bernoulli polynomials $B_{n}(x)$, and the generalized Bernoulli numbers $B_{n}(a, b)$.

2. Definitions and properties of generalized Bernoulli polynomials. It is easy to see that the following definition is a natural and essential generalization of the concepts of Bernoulli numbers $B_{n}$, Bernoulli polynomials $B_{n}(x)$, and the generalized Bernoulli numbers $B_{n}(a, b)$.

DEFINITION 2.1. Let $a, b, c>0$ and $a \neq b$. The generalized Bernoulli polynomials $B_{n}(x ; a, b, c)$ for nonnegative integer $n$ are defined by

$$
\phi(x ; t ; a, b, c)=\frac{t c^{x t}}{b^{t}-a^{t}}=\sum_{n=0}^{\infty} \frac{B_{n}(x ; a, b, c)}{n !} t^{n}, \quad|t|<\frac{2 \pi}{|\ln b-\ln a|}, x \in \mathbb{R} .
$$

The generalized Bernoulli polynomials $B_{n}(x ; a, b, c)$ have the following properties which are stated as theorems below.

THEOREM 2.2. Let $a, b, c>0$ and $a \neq b$. For $x \in \mathbb{R}$ and $n \geq 0$,

$$
\begin{aligned}
B_{n}(x ; 1, e, e) & =B_{n}(x), \quad B_{n}(0 ; a, b, c)=B_{n}(a, b) \\
B_{n}(0 ; 1, e, e) & =B_{n}, \quad B_{n}(x ; a, b, 1)=B_{n}(a, b), \quad B_{n}(x ; 1, e, 1)=B_{n} \\
B_{n}(x ; a, b, c) & =\sum_{k=0}^{n}\left(\begin{array}{l}
n \\
k
\end{array}\right)[\ln c]^{n-k} B_{k}(a, b) x^{x-k} \\
B_{n}(x ; a, b, c) & =\sum_{k=0}^{n}\left(\begin{array}{l}
n \\
k
\end{array}\right)[\ln c]^{n-k}[\ln b-\ln a]^{k-1} B_{k}\left(\frac{\ln a}{\ln a-\ln b}\right) x^{x-k} \\
B_{n}(x ; a, b, c) & =\sum_{k=0}^{n} \sum_{j=0}^{k}(-1)^{k-j}\left(\begin{array}{l}
n \\
k
\end{array}\right)\left(\begin{array}{l}
k \\
j
\end{array}\right)[\ln c]^{n-k}[\ln a]^{k-j}\left[\ln \frac{b}{a}\right]^{j-1} B_{j} x^{x-k} .
\end{aligned}
$$


Proof. Applying Definition 1.3 to the term $t /\left(b^{t}-a^{t}\right)$ and expanding the exponential function $c^{x t}$ at $t=0$ yields

$$
\begin{aligned}
\frac{t c^{x t}}{b^{t}-a^{t}} & =\left(\sum_{k=0}^{\infty} \frac{B_{k}(a, b)}{k !} t^{k}\right)\left(\sum_{i=0}^{\infty} \frac{x^{i}(\ln c)^{i}}{i !} t^{i}\right) \\
& =\sum_{k=0}^{\infty} \sum_{i=0}^{k} \frac{(\ln c)^{k-i}}{i !(k-i) !} B_{i}(a, b) x^{k-i} t^{k} \\
& =\sum_{n=0}^{\infty}\left(\sum_{k=0}^{n}\left(\begin{array}{l}
n \\
k
\end{array}\right)(\ln c)^{n-k} B_{k}(a, b) x^{n-k}\right) \frac{t^{n}}{n !} .
\end{aligned}
$$

Combining (2.6) and (2.1) and equating their coefficients of $t^{n}$ produces formula (2.3).

The following two formulae were provided in $[2,4]$ :

$$
\begin{aligned}
& B_{n}(a, b)=(\ln b-\ln a)^{n-1} B_{n}\left(\frac{\ln a}{\ln a-\ln b}\right), \\
& B_{n}(a, b)=\sum_{i=0}^{n}(-1)^{n-i}(\ln b-\ln a)^{i-1}(\ln a)^{n-i}\left(\begin{array}{c}
n \\
i
\end{array}\right) B_{i} .
\end{aligned}
$$

Substituting (2.7) and (2.8) into (2.3) leads to (2.4) and (2.5).

The formulae in (2.2) are obvious.

Now we give some results about derivatives and integrals of the generalized Bernoulli polynomials $B_{n}(x ; a, b, c)$ as follows.

THEOREM 2.3. Let $a, b, c>0, a \neq b, n \geq 0$, and $x \in \mathbb{R}$. For any nonnegative integer $\ell$ and real numbers $\alpha$ and $\beta$,

$$
\begin{aligned}
\frac{\partial^{\ell} B_{n}(x ; a, b, c)}{\partial x^{\ell}} & =\frac{n !}{(n-\ell) !}(\ln c)^{\ell} B_{n-\ell}(x ; a, b, c), \\
\int_{\alpha}^{\beta} B_{n}(t ; a, b, c) d t & =\frac{1}{(n+1) \ln c}\left[B_{n+1}(\beta ; a, b, c)-B_{n+1}(\alpha ; a, b, c)\right],
\end{aligned}
$$

where $B_{0}(x ; a, b, c)=1 /(\ln b-\ln a)$.

Proof. Formula (2.9) follows from standard arguments and induction.

Integrating on both sides of (2.9) with respect to variable $x$ for $\ell=1$ gives formula (2.10).

THEOREM 2.4. Let $a, b, c>0, a \neq b, n \geq 0$, and $x \in \mathbb{R}$. Then

$$
\begin{aligned}
& B_{n}(x+1 ; a, b, c)=\sum_{k=0}^{n}\left(\begin{array}{l}
n \\
k
\end{array}\right)(\ln c)^{n-k} B_{k}(x ; a, b, c), \\
& B_{n}(x+1 ; a, b, c)=B_{n}\left(x ; \frac{a}{c}, \frac{b}{c}, c\right),
\end{aligned}
$$


and, for $m \geq 2$,

$$
\begin{aligned}
B_{m}(x+1 ; a, b, c) & \\
= & B_{m}(x ; a, b, c)+m(\ln c)^{m-1} x^{m-1} \\
& +\sum_{k=0}^{m-1}\left(\begin{array}{c}
m \\
k
\end{array}\right)\left[(\ln a)^{m-k}-(\ln b)^{m-k}+(\ln c)^{m-k}\right] B_{k}(x ; a, b, c) .
\end{aligned}
$$

Proof. By the definition of the generalized Bernoulli polynomials, we have

$$
\begin{aligned}
\frac{t c^{(x+1) t}}{b^{t}-a^{t}} & =\sum_{n=0}^{\infty} \frac{B_{n}(x+1 ; a, b, c)}{n !} t^{n}, \\
\frac{t c^{(x+1) t}}{b^{t}-a^{t}} & =\frac{t c^{x t}}{b^{t}-a^{t}} \cdot c^{t} \\
& =\left(\sum_{n=0}^{\infty} \frac{B_{n}(x ; a, b, c)}{n !} t^{n}\right)\left(\sum_{k=0}^{\infty} \frac{(\ln c)^{k}}{k !} t^{k}\right) \\
& =\sum_{n=0}^{\infty} \frac{\sum_{k=0}^{n}\left(\begin{array}{l}
n \\
k
\end{array}\right)(\ln c)^{n-k} B_{k}(x ; a, b, c)}{n !} t^{n} .
\end{aligned}
$$

Combining (2.14) and (2.15) and equating their coefficients of $t^{n}$ leads to formula (2.11).

Similarly, since

$$
\frac{t c^{(x+1) t}}{b^{t}-a^{t}}=\frac{t c^{x t}}{(b / c)^{t}-(a / c)^{t}}=\sum_{n=0}^{\infty} \frac{B_{n}(x ; a / c, b / c, c)}{n !} t^{n},
$$

equating the coefficients of $t^{n}$ in (2.14) and (2.16) leads to formula (2.12).

Straightforward computation gives

$$
\begin{aligned}
\frac{t c^{(x+1) t}}{b^{t}-a^{t}}= & t c^{x t}+\frac{t c^{x t}\left(a^{t}-b^{t}+c^{t}\right)}{b^{t}-a^{t}} \\
= & \sum_{n=0}^{\infty} \frac{(\ln c)^{n} x^{n}}{n !} t^{n+1} \\
& +\left(\sum_{n=0}^{\infty} \frac{B_{n}(x ; a, b, c)}{n !} t^{n}\right)\left(\sum_{\ell=0}^{\infty} \frac{\left[(\ln a)^{\ell}-(\ln b)^{\ell}+(\ln c)^{\ell}\right]}{\ell !} t^{\ell}\right) \\
= & \sum_{n=0}^{\infty} \frac{(\ln c)^{n} x^{n}}{n !} t^{n+1} \\
& +\sum_{n=0}^{\infty}\left[\sum_{\ell=0}^{n}\left(\begin{array}{l}
n \\
\ell
\end{array}\right)\left[(\ln a)^{n-\ell}-(\ln b)^{n-\ell}+(\ln c)^{n-\ell}\right] B_{\ell}(x ; a, b, c)\right] \frac{t^{n}}{n !}
\end{aligned}
$$




$$
\begin{aligned}
= & B_{0}(x ; a, b, c)+\left[1+B_{1}(x ; a, b, c)+B_{0}(x ; a, b, c)(\ln a-\ln b+\ln c)\right] t \\
& +\sum_{n=2}^{\infty}\left[n(\ln c)^{n-1} x^{n-1}+B_{n}(x ; a, b, c)\right] \frac{t^{n}}{n !} \\
& +\sum_{n=2}^{\infty}\left\{\sum_{\ell=0}^{n-1}\left(\begin{array}{l}
n \\
\ell
\end{array}\right)\left[(\ln a)^{n-\ell}-(\ln b)^{n-\ell}+(\ln c)^{n-\ell}\right] B_{\ell}(x ; a, b, c)\right\} \frac{t^{n}}{n !} .
\end{aligned}
$$

Equating (2.1) and (2.17) yields (2.13).

COROLLARY 2.5. For $n \geq 1, b>0$, and $x \in \mathbb{R}$,

$$
B_{n}(x+1 ; 1, b, b)=B_{n}(x ; 1, b, b)+n(\ln b)^{n-1} x^{n-1} .
$$

REMARK 2.6. Taking $b=e$ in (2.18), the following well-known result is deduced:

$$
B_{n}(x+1)=B_{n}(x)+n x^{n-1}, \quad n \geq 1 .
$$

Similarly, from (2.9), it follows that

$$
B_{i}^{\prime}(t)=i B_{i-1}(t), \quad B_{0}(t)=1
$$

Actually, the Bernoulli polynomials $B_{i}(t), i \in \mathbb{N}$, are uniquely determined by formulae (2.19) and (2.20), see [1, identities 23.1.5 and 23.1.6] or [5].

TheOrem 2.7. Let $a, b, c>0, a \neq b, n \geq 0$, and $x \in \mathbb{R}$. Then

$$
\begin{aligned}
B_{n}(1-x ; a, b, c) & =(-1)^{n} B_{n}\left(x ; \frac{c}{b}, \frac{c}{a}, c\right) \\
& =B_{n}\left(-x ; \frac{a}{c}, \frac{b}{c}, \frac{1}{c}\right), \\
B_{n}(x+y ; a, b, c) & =\sum_{k=0}^{n}\left(\begin{array}{l}
n \\
k
\end{array}\right)(\ln c)^{n-k} B_{k}(x ; a, b, c) y^{n-k} \\
& =\sum_{k=0}^{n}\left(\begin{array}{l}
n \\
k
\end{array}\right)(\ln c)^{n-k} B_{k}(y ; a, b, c) x^{n-k} .
\end{aligned}
$$

Proof. From Definition 2.1, it follows that

$$
\frac{t c^{(1-x) t}}{b^{t}-a^{t}}=\sum_{n=0}^{\infty} \frac{B_{n}(1-x ; a, b, c)}{n !} t^{n}
$$


Meanwhile, we have

$$
\begin{aligned}
& \frac{t c^{(1-x) t}}{b^{t}-a^{t}}=\frac{t c^{-x t}}{(b / c)^{t}-(a / c)^{t}}=\sum_{n=0}^{\infty} \frac{B_{n}(-x ; a / c, b / c, c)}{n !} t^{n}, \\
& \frac{t c^{(1-x) t}}{b^{t}-a^{t}}=\frac{-t c^{x(-t)}}{(c / a)^{-t}-(c / b)^{-t}}=\sum_{n=0}^{\infty}(-1)^{n} \frac{B_{n}(x ; c / b, c / a, c)}{n !} t^{n} .
\end{aligned}
$$

Therefore, formula (2.21) follows from equating series expansions in (2.23) and (2.24).

Similarly, we have

$$
\begin{aligned}
\frac{t c^{(x+y) t}}{b^{t}-a^{t}} & =\sum_{n=0}^{\infty} \frac{B_{n}(x+y ; a, b, c)}{n !} t^{n}, \\
\frac{t c^{(x+y) t}}{b^{t}-a^{t}} & =\frac{t c^{x t}}{b^{t}-a^{t}} \cdot c^{y t} \\
& =\left(\sum_{n=0}^{\infty} \frac{B_{n}(x ; a, b, c)}{n !} t^{n}\right)\left(\sum_{i=0}^{\infty} \frac{y^{i}(\ln c)^{i}}{i !} t^{i}\right) \\
& =\sum_{n=0}^{\infty}\left(\sum_{k=0}^{n}\left(\begin{array}{l}
n \\
k
\end{array}\right) y^{n-k}(\ln c)^{n-k} B_{k}(x ; a, b, c)\right) \frac{t^{n}}{n !}, \\
\frac{t c^{(x+y) t}}{b^{t}-a^{t}} & =\frac{t c^{y t}}{b^{t}-a^{t}} \cdot c^{x t} \\
& =\sum_{n=0}^{\infty}\left(\sum_{k=0}^{n}\left(\begin{array}{l}
n \\
k
\end{array}\right) x^{n-k}(\ln c)^{n-k} B_{k}(y ; a, b, c)\right) \frac{t^{n}}{n !} .
\end{aligned}
$$

Hence, formula (2.22) follows from equating series expansions in (2.25). The proof is complete.

THEOREM 2.8. Let $m$ and $n$ be natural numbers. Then, for any positive number $b$, the following identity holds:

$$
\begin{aligned}
\sum_{j=1}^{m} j^{n} & =\frac{1}{(n+1)(\ln b)^{n}}\left[B_{n+1}(m+1 ; 1, b, b)-B_{n+1}(0 ; 1, b, b)\right] \\
& =\frac{1}{(n+1)(\ln b)^{n}}\left[B_{n+1}(m+1 ; 1, b, b)-B_{n+1}(1 ; 1, b, b)\right] .
\end{aligned}
$$

PROoF. Rewriting formula (2.18) yields

$$
x^{n-1}=\frac{1}{n(\ln b)^{n-1}}\left[B_{n}(x+1 ; 1, b, b)-B_{n}(x ; 1, b, b)\right],
$$


which implies

$$
j^{n}=\frac{1}{(n+1)(\ln b)^{n}}\left[B_{n+1}(j+1 ; 1, b, b)-B_{n+1}(j ; 1, b, b)\right] .
$$

Summing up on both sides of (2.28) from 0 to $m$ or from 1 to $m$ with respect to $j$ easily leads to formula (2.26).

REMARK 2.9. The calculation of values of $\sum_{j=1}^{m} j^{n}$ is an interesting problem that has been investigated in many works, see, for example, [3].

REMARK 2.10. It follows from the identities (2.3) and (2.7), combined with [1, identity 23.1.7], that

$$
B_{n}(x ; a, b, c)=(\ln b-\ln a)^{n-1} B_{n}\left(\frac{\ln a-x \ln c}{\ln a-\ln b}\right) .
$$

REMARK 2.11. At last, it is pointed out that the Bernoulli and Euler numbers and the Bernoulli and Euler polynomials can be further generalized to more general results in this manner. These conclusions will be published in some subsequent papers.

ACKNOWLEDGments. This note was finalized during the third author's visit to the RGMIA with grants from the Victoria University and Jiaozuo Institute of Technology. The authors would like to express many thanks to the anonymous referee for many valuable comments and suggestions. The first three authors were supported in part by NNSF of China, Grant 10001016, SF for the Prominent Youth of Henan Province, Grant 0112000200, SF of Henan Innovation Talents at Universities, NSF of Henan Province, Grant 004051800, SF for Pure Research of Natural Science of the Education Department of Henan Province, Grant 1999110004, Doctor Fund of Jiaozuo Institute of Technology, China. The fourth author was partially supported by a grant of the Faculty Research Council of the University of Texas-Pan American.

\section{REFERENCES}

[1] M. Abramowitz and I. A. Stegun (eds.), Handbook of Mathematical Functions, with Formulas, Graphs, and Mathematical Tables, 3rd ed., with corrections, National Bureau of Standards, Applied Mathematics Series, vol. 55, US Government Printing Office, Washington, D.C., 1965.

[2] B.-N. Guo and F. Qi, Generalization of Bernoulli polynomials, Internat. J. Math. Ed. Sci. Tech. 33 (2002), no. 3, 428-431.

[3] S.-L. Guo and F. Qi, Recursion formulae for $\sum_{m=1}^{n} m^{k}$, Z. Anal. Anwendungen 18 (1999), no. 4, 1123-1130.

[4] F. Qi and B.-N. Guo, Generalisation of Bernoulli polynomials, RGMIA Res. Rep. Coll. 4 (2001), no. 4, Article 10, 691-695, http://rgmia.vu.edu.au/v4n4.html.

[5] Zh.-X. Wang and D.-R. Guo, Introduction to Special Function, The Series of Advanced Physics of Peking University, Peking University Press, Beijing, 2000 (Chinese). 
Qiu-Ming Luo: Department of Broadcast-Television Teaching, Jiaozuo University, Jiaozuo City, Henan 454002, China

E-mail address: 1uoqm@jzu.edu.cn

Bai-Ni Guo: Department of Applied Mathematics and Informatics, Jiaozuo Institute of Technology, Jiaozuo City, Henan 454000, China

E-mail address: guobaini@jzit.edu.cn

Feng Qi: Department of Applied Mathematics and Informatics, Jiaozuo Institute of Technology, Jiaozuo City, Henan 454000, China

E-mail address: qi feng@jzit.edu.cn

URL: http://rgmia.vu.edu.au/qi .html

Lokenath Debnath: Department of Mathematics, University of Texas-Pan American, Edinburg, TX 78539, USA

E-mail address: debnath1@panam.edu 


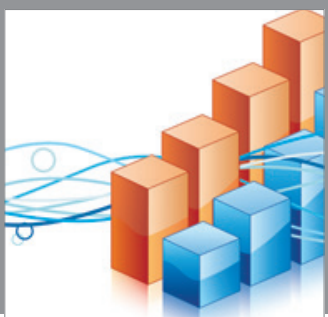

Advances in

Operations Research

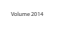

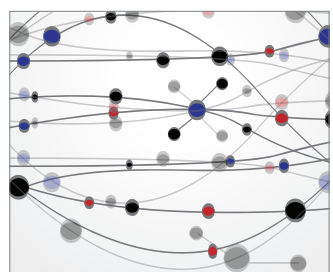

\section{The Scientific} World Journal
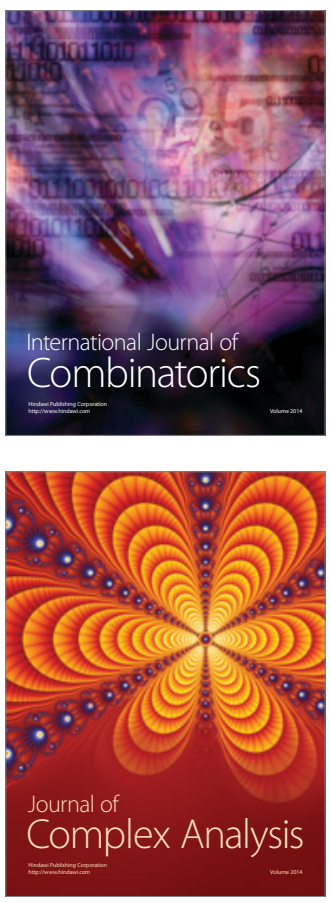

International Journal of

Mathematics and

Mathematical

Sciences
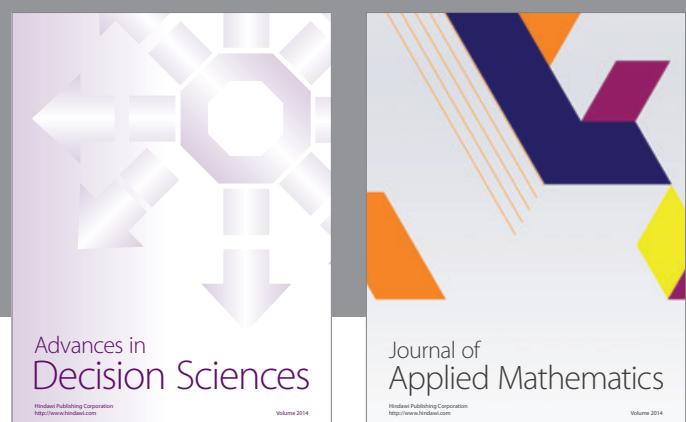

Journal of

Applied Mathematics
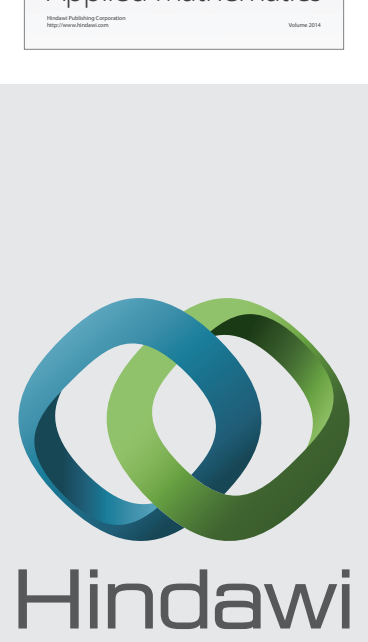

Submit your manuscripts at http://www.hindawi.com
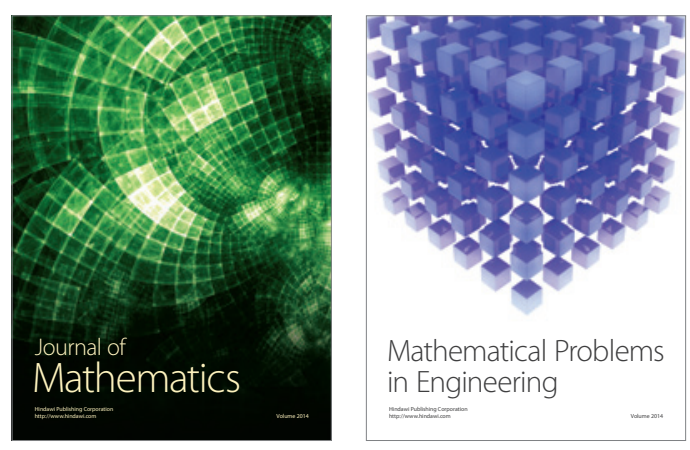

Mathematical Problems in Engineering
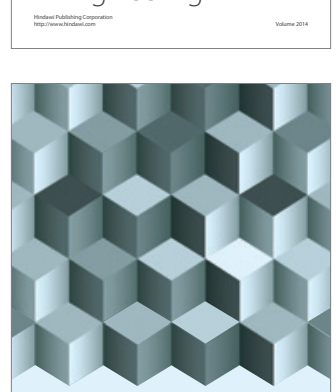

Journal of

Function Spaces
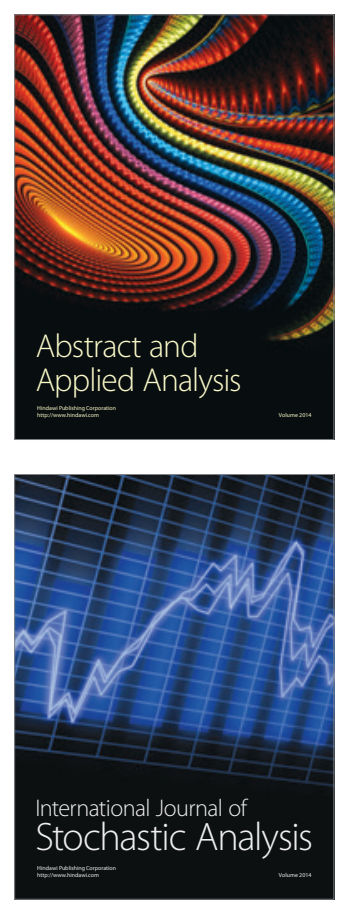

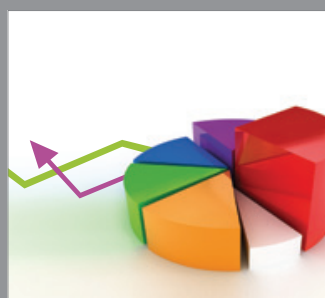

ournal of

Probability and Statistics

Promensencen
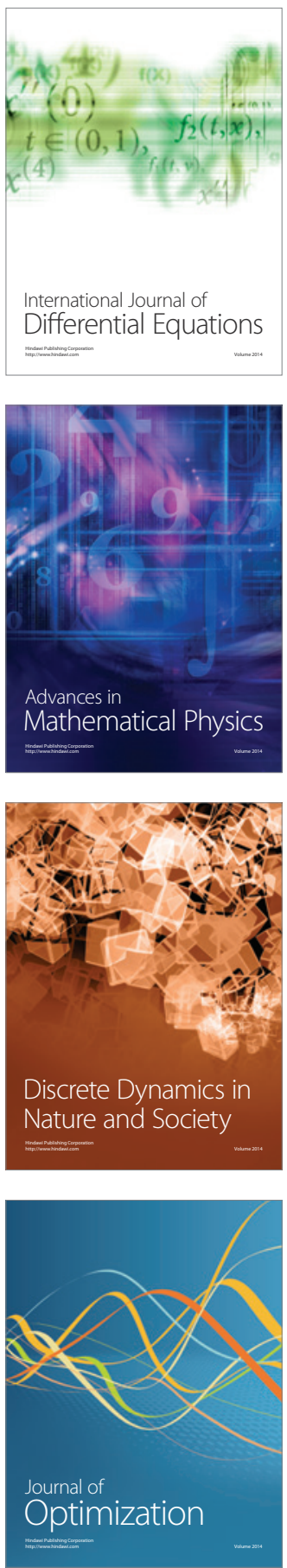